\title{
Self-Reports of Potentially Traumatic Experiences in an Adult Community Sample: Gender Differences and Test-Retest Stabilities of the Items in a Brief Betrayal-Trauma Survey
}

\author{
Lewis R. Goldberg, $\mathrm{PhD}$ \\ Jennifer J. Freyd, PhD
}

\begin{abstract}
A new survey of potentially traumatic events was administered to a large community sample on two occasions, three years apart. In contrast to previous surveys, this one included separate items for events that involve mistreatment by someone close, mistreatment by someone not so close, and non-interpersonal events. For both kinds of
\end{abstract}

Lewis R. Goldberg is Senior Scientist at Oregon Research Institute and Jennifer J. Freyd is Professor of Psychology at the University of Oregon, OR.

Address correspondence to: Lewis R. Goldberg, Oregon Research Institute, 1715 Franklin Boulevard, Eugene, OR 97403-1983 (E-mail: lewg@ori.org).

The authors are indebted to Carolyn Allard, Michael Anderson, Michael Ashton, M. Rose Barlow, Kathy Becker, Lisa D. Cromer, Anne DePrince, Rachel Goldsmith, Christopher Graver, Sarah E. Hampson, Alice King, Bridget Klest, Del Paulhus, Gerard Saucier, Sanjay Srivastava, and Eileen Zurbriggen for many thoughtful comments and suggestions.

This research was supported by Grant MH49227 to Dr. Lewis R. Goldberg from the National Institute of Mental Health. The writing of this paper was facilitated by funds for Dr. Jennifer J. Freyd from the Trauma and Oppression Research Fund of the University of Oregon Foundation.

The action editor for this article was Laura S. Brown, PhD ABPP, Associate Editor

Because the BBTS is continually undergoing refinement, we post the most current version at an Internet website; readers interested in this instrument can obtain it at: http://dynamic.uoregon.edu/ jjf/bbts/

Journal of Trauma \& Dissociation, Vol. 7(3) 2006

Available online at http://www.haworthpress.com/web/JTD

(c) 2006 by The Haworth Press, Inc. All rights reserved.

doi:10.1300/J229v07n03_04 
interpersonal events, separate items focused on physical, sexual, and emotional types of potential abuse. For each event, respondents indicated the extent of their exposure both prior to and after age 18. This paper reports the prevalence of each of the various kinds of events in subsamples of women $(\mathrm{N}=397)$ and men $(\mathrm{N}=292)$ in both childhood and adulthood, and provides four alternative indices of test-retest stability for each of the event reports. Substantial differences between men and women were found for many of the reported events on both occasions. Specifically, far more women than men reported having experienced traumatic events perpetrated by someone close to them, whereas far more men than women reported having experienced traumatic events perpetrated by someone not close. Some of the implications of these gender interaction effects are discussed. [Article copies available for a fee from The Haworth Document Delivery Service: 1-800-HAWORTH. E-mail address: <docdelivery@haworthpress.com>Website: <http://www.HaworthPress. com> (c) 2006 by The Haworth Press, Inc. All rights reserved.]

KEYWORDS. Traumatic event prevalence, traumatic event retest stability, abuse, gender differences, brief betrayal-trauma survey

Certainly one of the major tasks of developmental psychology is to discover the subsequent effects of significant life events, especially experiences that occur early in life and particularly those that are disturbing, perhaps even traumatic, in their later impact. Because of the enormous difficulty in recording events at the time of their occurrence, investigators typically must rely for their information on retrospective self-reports. However, as is well known, there are many potential limitations to an individual's ability and/or willingness to report the details of intimate life events. As a consequence, one of the great scientific challenges for research on psychopathology is that of developing reliable and valid measures of event occurrences.

In this paper, we address that important scientific challenge by reporting findings based on a new and still-evolving measure of event occurrence. In a brief overview, on the basis of a theory of event impact, we developed a 12-event survey that was administered to a large community sample in 2000. Then, based on the findings from extensive analyses of the responses to that initial survey, we developed a new 14-event survey (with additional multiple-choice and open-ended questions) which we administered to the same sample in 2003. In this paper, we describe the rationale for the development of these instruments, and we examine the 
event frequencies on each administration, separately for men and women, as well as the test-retest stabilities of the event reports over the three-year interval. Finally, we discuss the implications of these and other findings for theories linking traumatic events to later life outcomes.

Survey-based research on the prevalence of potentially traumatic events in the United States has generally revealed high levels of lifetime exposure. Norris (1992) administered a survey to 1,000 individuals in four southeastern cities; in that study, $69 \%$ reported experiencing at least one traumatic event. In a mailed questionnaire study by Elliott (1997), $72 \%$ of the 505 participants reported some form of major traumatic event, $40 \%$ experienced a major motor-vehicle accident or natural disaster, $43 \%$ witnessed interpersonal violence, $50 \%$ were victims of such violence, and $23 \%$ reported childhood sexual abuse. Within the last decade, many other investigators, using a variety of instruments and measurement techniques, have reported comparable rates of traumatic event exposure (see Carlson, 1997; National Center for PTSD, 2006; Wilson $\&$ Keane, 2004).

\section{THEORETICAL RATIONALE}

Although there is widespread agreement that some traumatic events can leave an enduring psychological impact, little is known about the relations between the particular characteristics of such events and individuals' psychological reactions to them. Do all such events potentially lead to the same sort of symptoms, perhaps varying in degree but not in kind? If so, what causes some types of events to lead to more distress than others? Or, are there different kinds of harm associated with different kinds of potentially disturbing events?

According to betrayal-trauma theory (Freyd, 1994, 1996), experiences involving a betrayal of trust, such as childhood abuse perpetrated by an adult who is quite close to the victim, led to a set of outcomes that differ in kind from traumas that do not involve betrayal. Freyd (1999, 2001) hypothesized that separate clusters of symptoms of post-traumatic distress arise from two distinct dimensions of harm-life threat and social betrayal. Life threat is predicted to lead to symptoms of anxiety and hyper-arousal; social betrayal should lead to symptoms of dissociation, emotional numbness and depression, and constricted or abusive relationships. High levels of both life threat and social betrayal characterize the most severe traumatic events; with both aspects present, both classes of symptoms can co-occur, as in cases of post-traumatic stress 
disorder. In summary, betrayal-trauma theory emphasizes the nature of the relationship between the victim and the perpetrator, particularly whether or not the perpetrator is a caregiver. In that crucial case, dissociative responses such as forgetting are predicted to be adaptive. In order to test this core theoretical prediction, Freyd (1996) reanalyzed a number of data sets and found that memories for incest were more likely to be lost and later recovered than were memories for other forms of childhood abuse.

\section{THE INITIAL DEVELOPMENT OF A BRIEF BETRAYAL-TRAUMA SURVEY}

Freyd and her colleagues have developed a measure of event history, the Betrayal Trauma Inventory (BTI), which provides detailed information about the relationship between the victim and the perpetrator, as well as many other characteristics of each event. Based upon some preliminary findings with the BTI, Freyd, DePrince, and Zurbriggen (2001) reported that sexual and physical abuse perpetrated by a victim's caregiver, when compared with abuse perpetrated by others, was related to reports of less persistent memories of the abuse. These findings are consistent with betrayal-trauma theory, and the BTI should be useful for testing additional hypotheses related to that theory.

However, the BTI takes approximately 45 minutes for participants to complete, and consequently it is not practical for use in studies in which many other instruments or experimental procedures must be included. Furthermore, the BTI involves detailed and probing questions, at least some of which might be considered intrusive or disturbing by many of the research participants in community samples. But, if interpersonal betrayal-related events are especially potent for producing particular sorts of psychological reactions, then it is important to be able to measure the occurrence and frequency of such events in a convenient format. Although many measures of trauma history have recently become available (see Norris, 1992; Norris \& Riad, 1997; Wilson \& Keane, 2004), the field has lacked a brief instrument that discriminates experiences with betrayal-related events (interpersonal events in a close relationship) from other kinds of potentially traumatic events.

In creating the instrument, we were guided by two goals: (1) to include as many as possible of the major kinds of potentially traumatic events and (2) to keep the instrument as short as possible. Because these two goals work in opposition to each other, we focused on four basic 
types of event discriminations: (1) Interpersonal from non-interpersonal events, (2) betrayal events (where the perpetrator had a close relationship with the target) from other interpersonal events (where the relationship was not so close), (3) childhood from adult events, and (4) physical versus sexual versus emotional types of abuse. In developing the items, we tried to use neutral language emphasizing specific behaviors ("touching," "penetration," "bruises," and "made to have sexual contact"), and to avoid stigmatizing labels ("raped"). Finally, we tried to insure that the final instrument would be amenable to administer to participants in an adult community sample, some of whom would come from backgrounds where intimate and/or sexual behaviors are not normally discussed.

Starting with the items included in the BTI, the second author compiled an initial set of items, which was then revised and edited by the first author. In an iterative fashion, the item pool was revised in response to the second author's pressure to be as specific as possible in the wording of the item content and the first author's sensitivity to potential concerns on the part of the research participants. Eventually we reached agreement on an initial set of 12 events. To test the psychometric characteristics of these items, we administered them to the participants of an ongoing community sample, about whom extensive personality and demographic information was already available.

\section{Participants}

All of the research participants were members of the Eugene-Springfield Community Sample (ESCS), which was recruited by a mail solicitation in 1993 from lists of local homeowners. The participants are remarkably heterogeneous on most variables other than racial/ethnic status (virtually all of them are Caucasian), ranging in age from 18 to 85 in 1993. All levels of educational attainment are represented in the sample, the average participant having completed about two years of post-secondary schooling. Over the past 11 years, the participants have been administered about two questionnaires a year by mail, covering a wide variety of measures of personality attributes, health practices and attitudes, and symptoms associated with many kinds of mental disorders; for further details about the ESCS, see Goldberg (1999a, 1999b). In a 16-page questionnaire administered to the ESCS in 2000, one page included the 12 events listed here in the Appendix. Of the roughly 850 participants who were sent copies of the questionnaire, 749 (432 women 
and 317 men) returned them. Of these, 726 (422 women and 304 men) provided information about all of the events.

\section{Revising the Initial Survey}

The questionnaire included three pairs of items that were worded almost identically except that one included the phrase "someone with whom you were very close (such as a parent, brother, sister, caregiver, or intimate partner)" and the other included the phrase "someone with whom you were not so close": (Items 3/4) Witnessed [that person] committing suicide, being killed or being injured by another person so severely as to result in marks, bruises, blood, or broken bones; (Items 5/6) You were deliberately attacked that severely by [that person]; and (Items 7/8) You were made to have some form of sexual contact, such as touching or penetration by [that person]. Our analyses of the responses to these three event pairs, which we will report later in this paper, showed substantial interactions between gender and the "very close" versus the "not very close" versions of the same events. As a consequence, in revising our survey we added two new items to cover the "not very close" option for those two events for which only the "very close" version had been included in the initial survey: (Item 5) Witnessed [that person] deliberately attack another family member so severely as to result in marks, bruises, blood, broken bones, or broken teeth; and (Item 10) You were emotionally or psychologically mistreated over a significant period of time by [that person].

Moreover, our analyses of the initial survey suggested that we should include some probes for additional information. First, we discovered that many participants reported having experienced "a seriously traumatic event" not included among our initial set, which we had assumed to be reasonably comprehensive in scope. Consequently, we added an openended question asking the participants to describe "any seriously traumatic" events that had not been included. Later in this paper, we will report on the frequencies of the types of events that were listed under this option. And, finally, for each of the events that were reported to have occurred, we asked participants to provide information about the gender of the perpetrator and the nature of their relationship (parent or guardian; other family member; husband, wife, or romantic partner; babysitter or nanny; teacher, doctor, coach, religious counselor, or other professional; friend or acquaintance; stranger), in order to discover whether men and women differ in their classifications into the categories "very close" and "not very close." (Future versions might profitably replace "other profes- 
sional" with "other professional or organizational leader.") Thus, the second survey provided information permitting us to disentangle actual gender differences in reported experiences from possible gender differences in the classification process of "close" and "not close."

This second version of the Brief Betrayal-Trauma Survey (BBTS) was administered to the community sample in 2003, three years after the first version. Of the 412 women in the sample who completed the second version of the BBTS, 397 (96\%) had also completed the initial survey; of the 321 men who completed the second version, 292 (91\%) had also completed the first survey. Thus, in the present paper we present the findings from our analyses of the 397 women and 292 men who completed the BBTS on both occasions.

\section{RESULTS}

\section{The Reported Frequencies of the Events}

Table 1 presents the percentages of men and of women who reported having experienced each of the events, both before and after 18 years of age, which for convenience we will refer to as "childhood" and "adulthood." For comparison purposes, the relative frequencies of each event are presented both for the first administration of the BBTS in 2000 (the upper value in each cell of the table) and the second administration in 2003 (the lower value). The events are classified by their type (Non-interpersonal, Interpersonal, Close Personal, and Other).

The events varied enormously in the proportion of participants who reported having experienced them, ranging from nearly zero (the death of one's child during one's own childhood) to nearly $50 \%$ (women reporting some other [non-specified] seriously traumatic event in adulthood on the first administration of the survey). When one compares the event frequencies between the two occasions, the two frequencies in each such pair were within a few percentages of each other with the exception that a substantially smaller percentage of women now reported experiencing some other [non-specified] seriously traumatic event in adulthood on the second administration of the survey.

\section{Gender Differences in the Event Frequencies}

Included in Table 1 are the percentage differences between men and women in their reports of event occurrences, and the statistical signifi- 


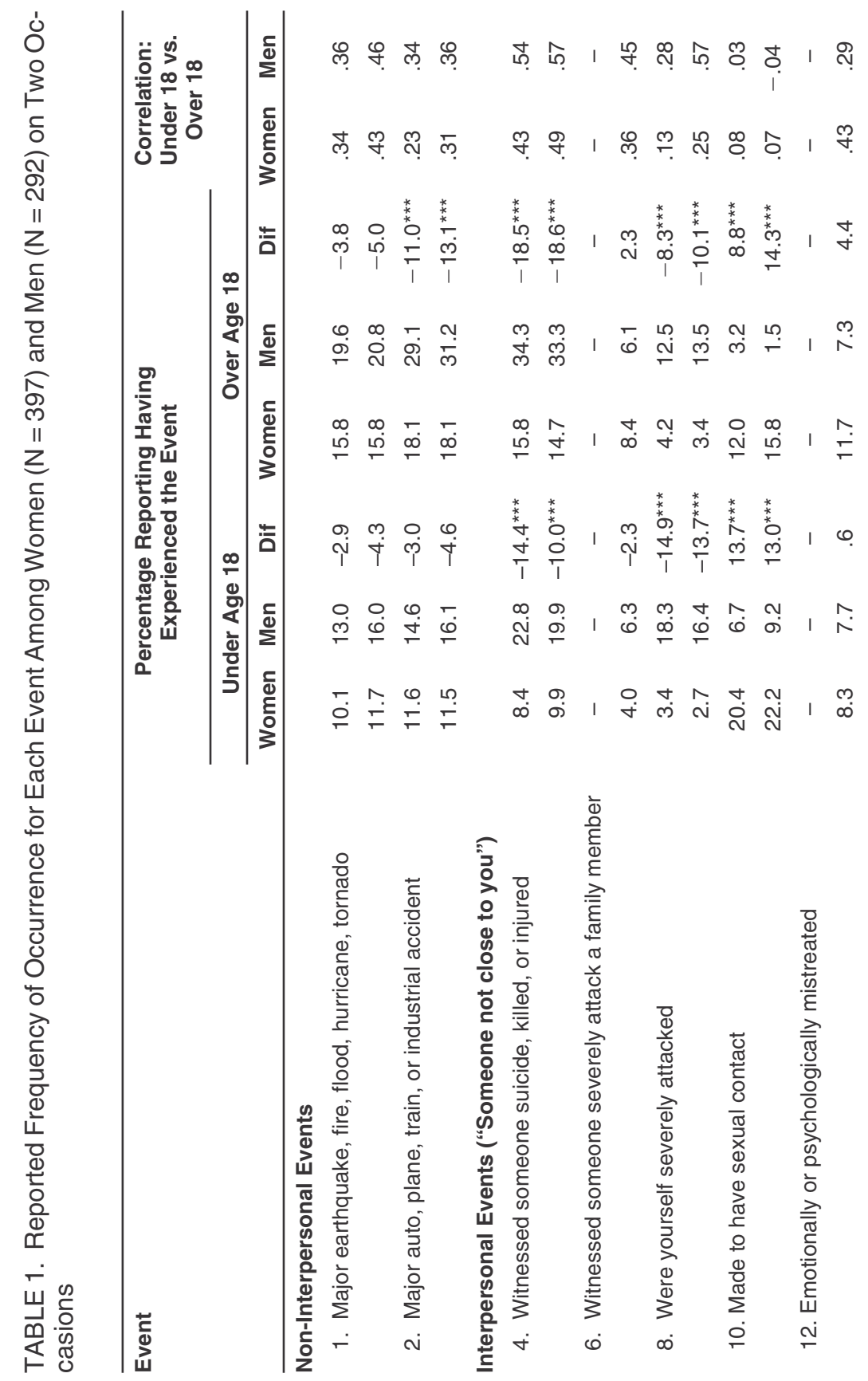




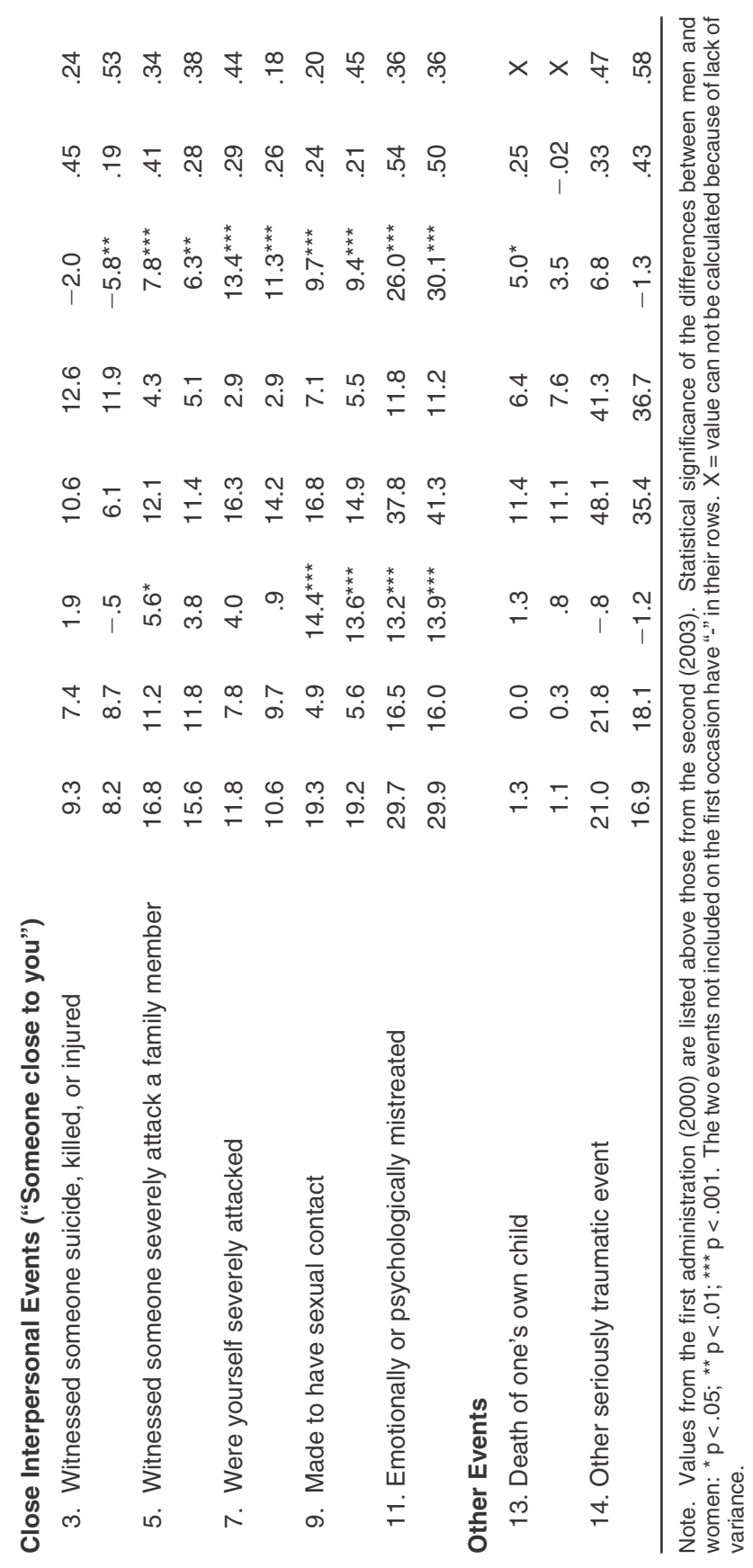


cance of these gender effects. Substantially more women than men reported having experienced emotional or psychological mistreatment by someone close, both as adults (around $40 \%$ vs. less than 12\%) and as children (around $30 \%$ vs. 16\%). Moreover, considerably more women than men reported both childhood and adult sexual abuse (whether perpetrated by someone close or not close to them). On the other hand, substantially more men than women reported having witnessed someone not close to them being killed, committing suicide, or being severely injured, both in childhood and as adults. In general, men reported having experienced more non-interpersonal events and events involving someone not close to them, whereas women reported having experienced more events involving someone close to them.

Perhaps the best way to illustrate this interaction between gender and type of relationship is by comparing the relative frequencies for the two events not involving sex abuse from the first survey that were described identically except for the substitution of the phrase "someone with whom you were very close" for the phrase "someone with whom you were not so close." Figure 1 shows these frequencies, separately for each of the two events in both childhood and adulthood. Figure 2 provides the first administration percentages of respondents of each gender who reported having experienced at least one event of varying degrees of potential trauma, ranging from none at all, to events of low potential trauma (e.g., natural disasters, accidents, witnessing someone not close getting hurt), to events of medium potential trauma (e.g., witnessing the death of someone not close, being severely attacked by someone not close), to events that are most likely to elicit betrayal traumas (e.g., being attacked by someone close, being made to have sex by someone close, being mistreated by someone close). We will examine these gender differences, and discuss their theoretical implications, more extensively later in this paper.

\section{Reliability of the Event Reports Across the Two Occasions}

How stable were these self-reports across the three-year period between the two administrations of the BBTS? The four panels of Table 2 provide the relevant test-retest data separately for men and women, in childhood and in adulthood, for each of the 12 events that were included in both surveys. Included in the table are the proportions of responses of each of nine types (N-N, Y-Y, N-Y, Y-N, M-M, N-M, Y-M, M-N, and $\mathrm{M}-\mathrm{Y}$ ), where $\mathrm{N}=\mathrm{No}$ (the event did not occur), $\mathrm{Y}=\mathrm{Yes}$ (the event did occur), and $\mathrm{M}=$ Missing data, from the first to the second administra- 
FIGURE 1. Percentages for the pairs of BBTS items not involving sex abuse that were worded identically except for the substitution of the phrase "someone with whom you were very close" for the phrase "someone with whom you were not so close." Percentages are presented separately for childhood (left) and adulthood (right).
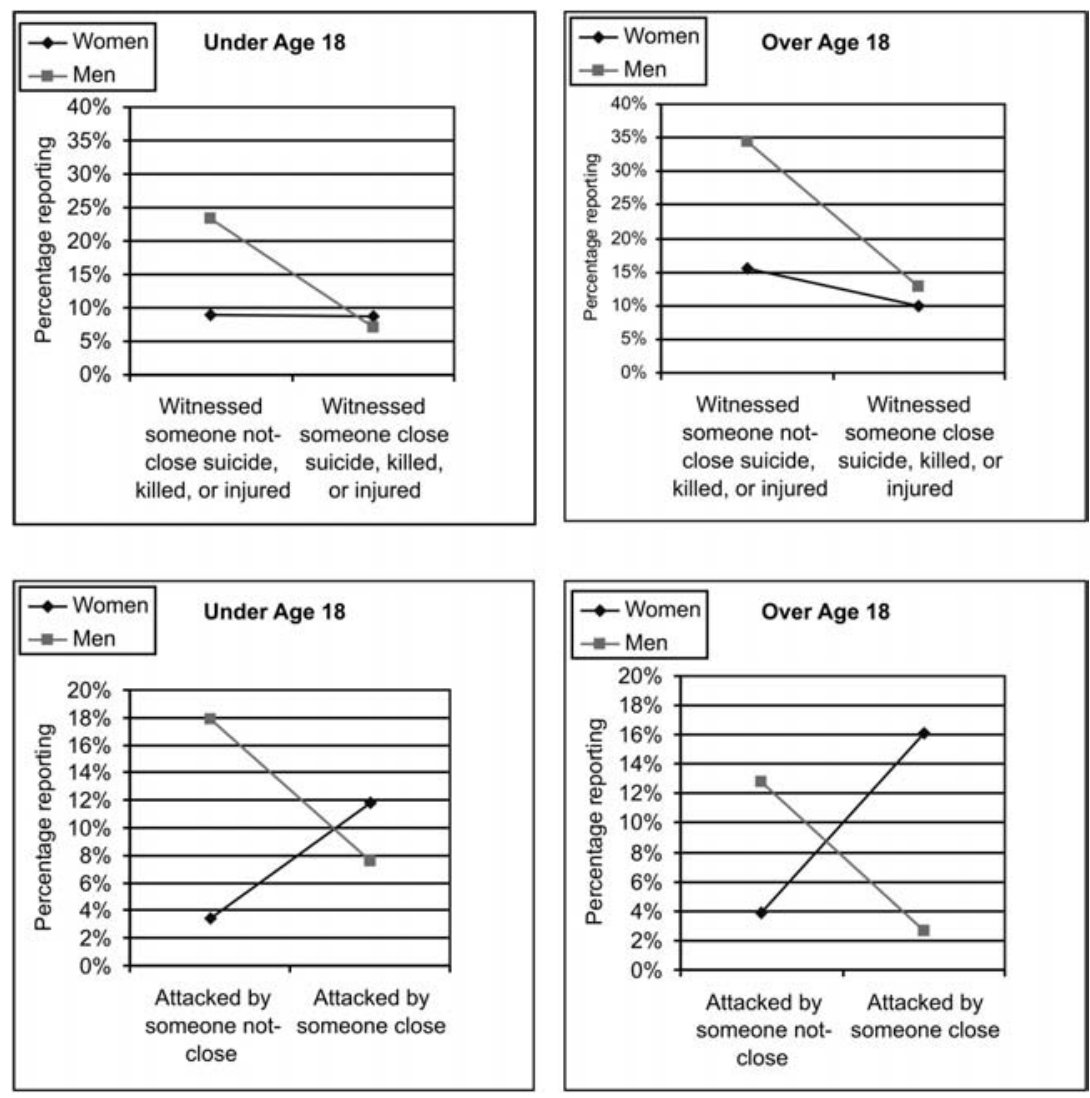

tion, respectively. The first four columns provide frequencies for those individuals who gave Yes or No responses on both occasions, and thus these columns are useful for analyses in which missing data are discarded. The remaining five columns in the table provide the relative frequencies for individuals who did not respond to that event on one or both of the two occasions. 
FIGURE 2. The percentages of respondents of each gender who reported having experienced at least one event of varying degrees of potential trauma, ranging from none at all, to events of low potential trauma (e.g., natural disasters), to events of medium potential trauma (e.g., witnessing the death of someone not close), to events that are most likely to elicit betrayal traumas (e.g., being attacked by someone close). Subjects are categorized by their most traumatic reported event. High betrayal: items 6, 8, and/or 10 (from Table 1); Medium betrayal: items 3, 5, 7, 9, 11, and/or 12; Low betrayal: items 1, 2, and/or 4.
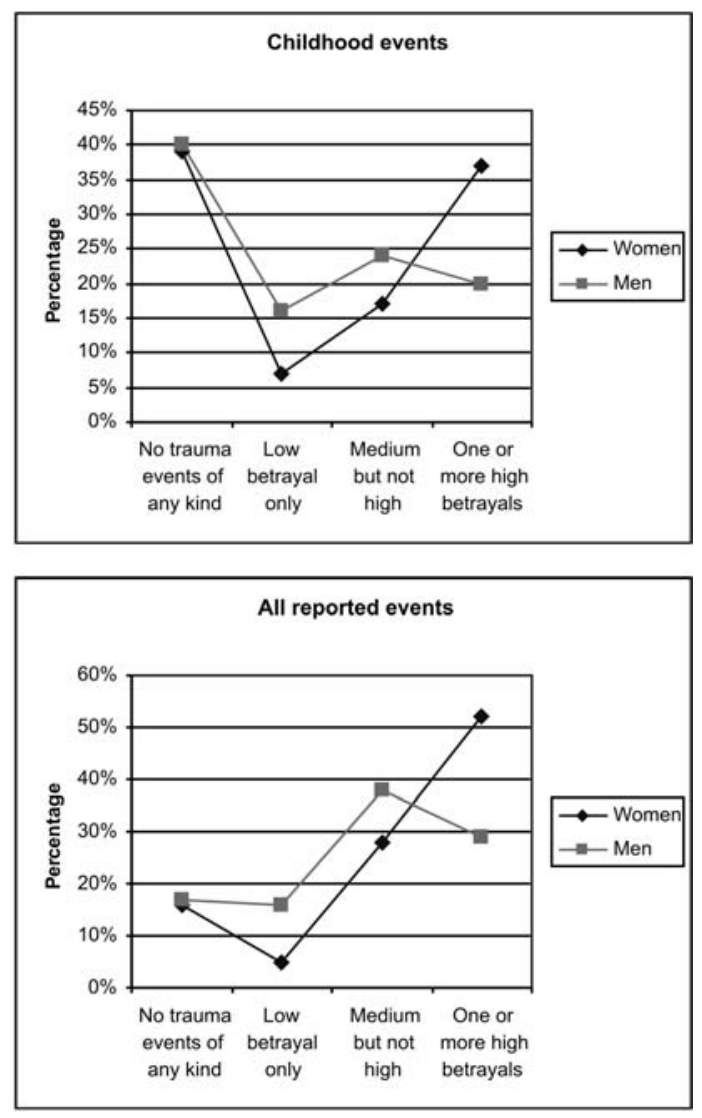

How is one to consider such missing data in analyzing the stability of the event reports? Some readers may be prone to equate missing data with "No" responses (perhaps on the grounds that both involve the absence of a positive response), whereas other readers may suspect that missing data 


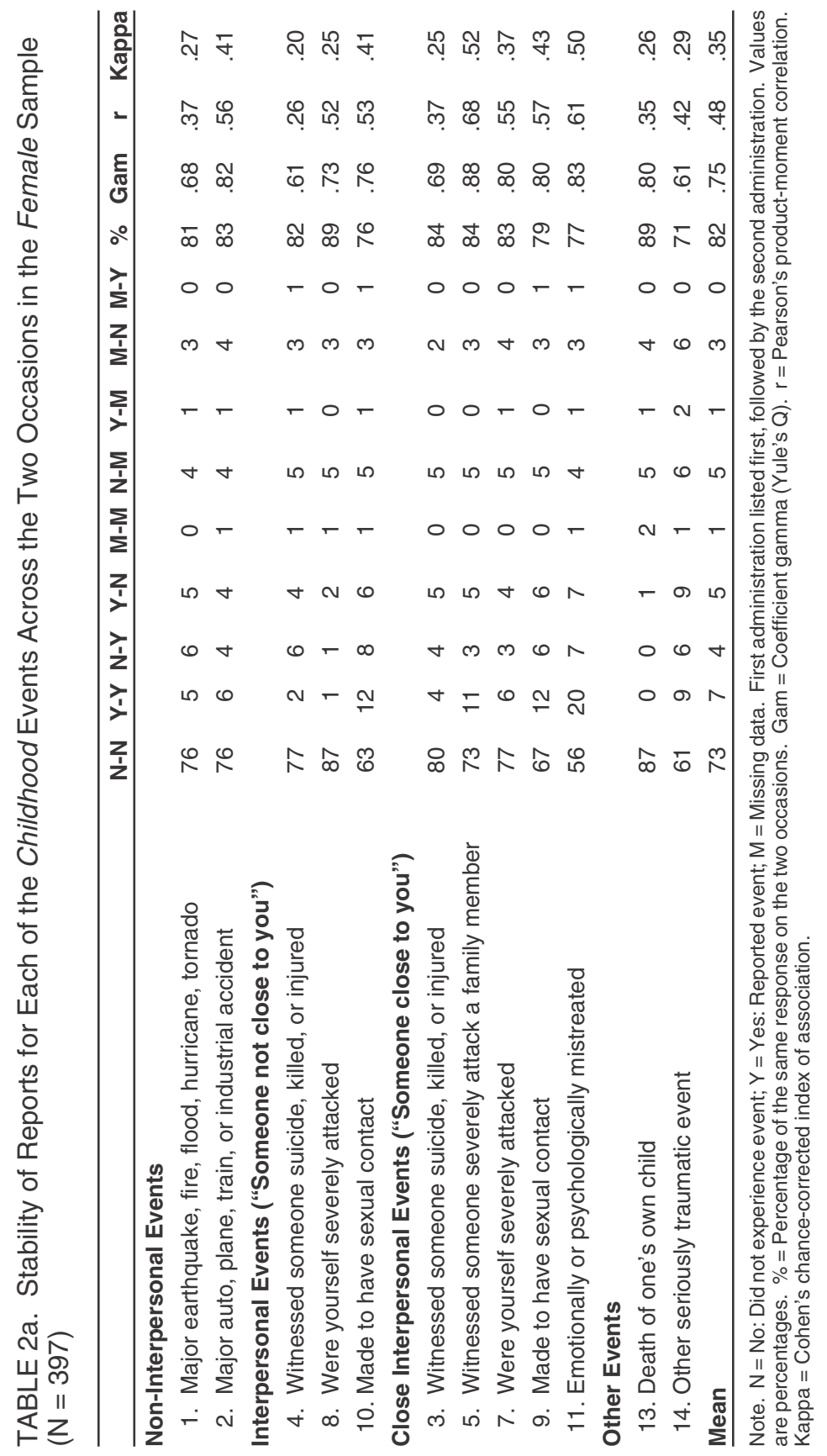




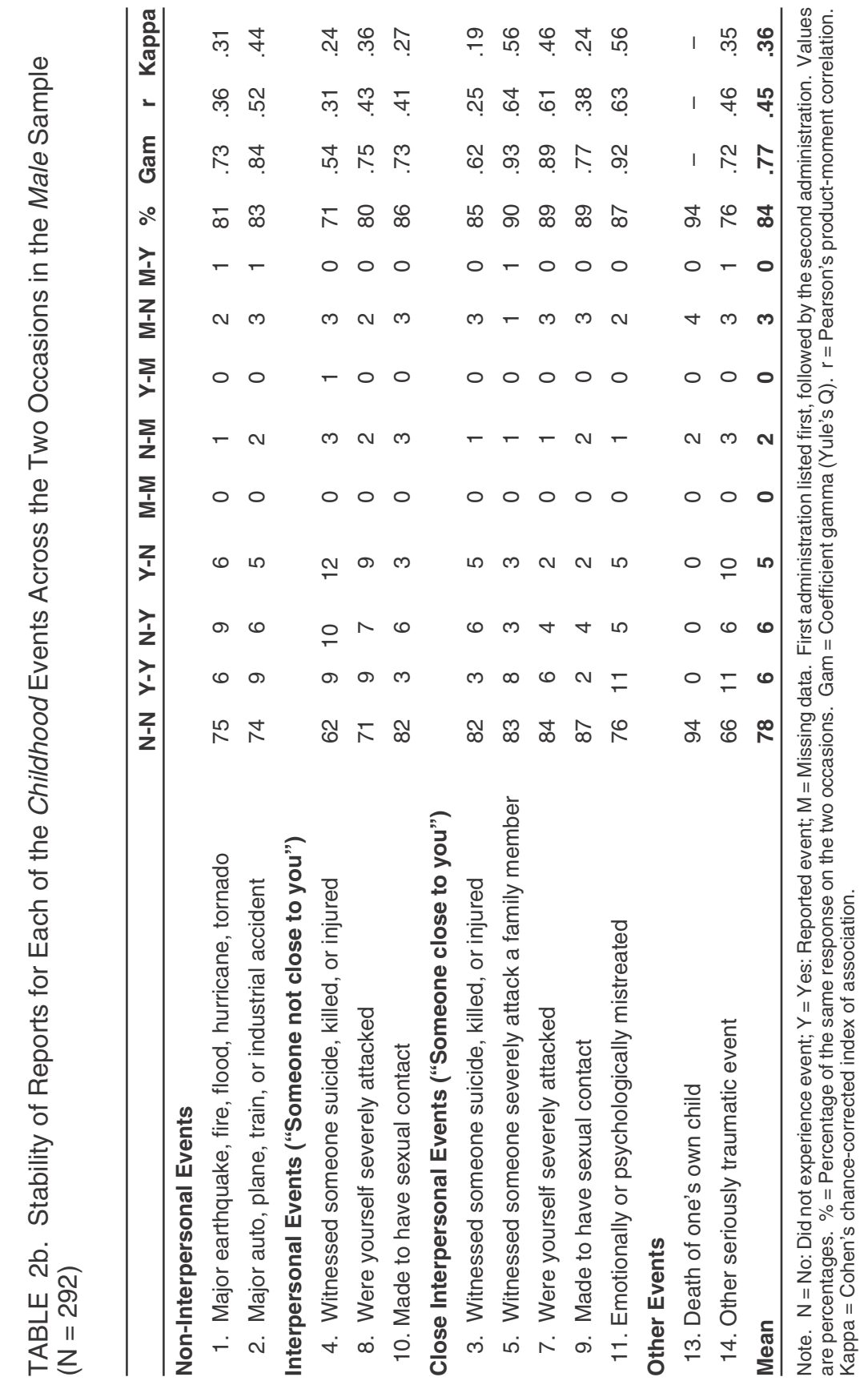




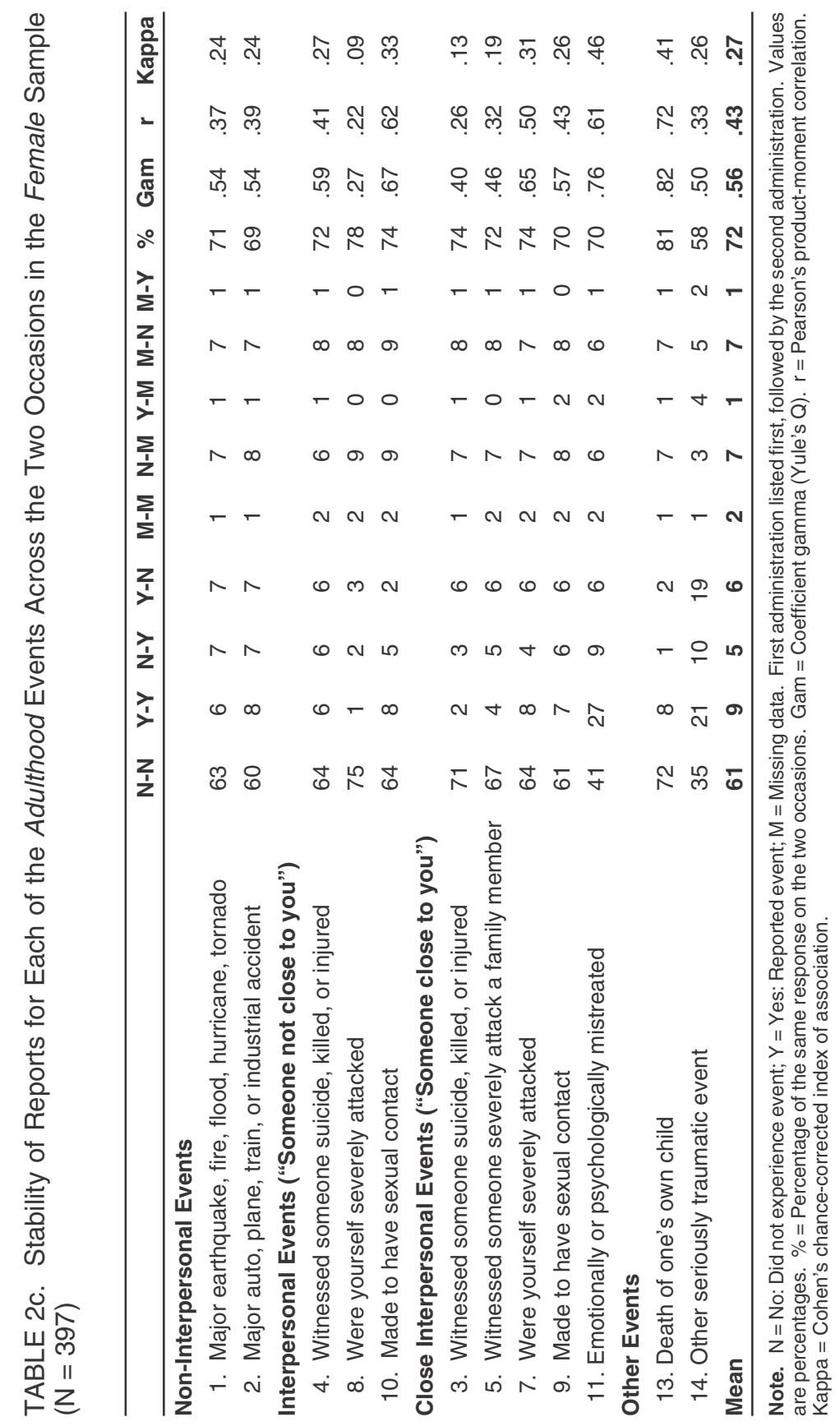







are more akin to "Yes" responses (perhaps from individuals who are reticent to make such a claim). Although we suspect that both possibilities may exist, we feel more comfortable relying on standard psychometric theory, which posits that missing answers tend to reflect lack of knowledge (akin to "I don't know" or "I'm not sure" in answering a question). On balance, then, we assume that missing data are best conceived as lying somewhere in the middle region between Yes and No responses. Treated in that way, we can reconceptualize each event response from a dichotomous (Yes vs. No) to a trichotomous (Y vs. M vs. N) format.

One advantage of that procedure is that when the responses from two administrations of the same event are analyzed together, it provides a more differentiated response scale for the analysis of test-retest data than the traditional two-by-two table. Specifically, we can now analyze the relative frequency of each event on a latent continuum that runs from (1) Yes on both occasions, (2) Yes-Missing or Missing-Yes, (3) Yes-No or Missing on both occasions, (4) No-Missing or Missing-No, and finally (5) No on both occasions. Using this metric, we have provided in Table 2 four indices of test-retest stability: (1) The percentage agreement (the percentage of the same response on the two occasions), (2) Coefficient gamma (Yule's Q), (3) r (the Pearson product-moment correlation), and (4) Kappa (Cohen's chance-corrected index of association). Of these four indices of association, the least familiar is probably the gamma coefficient, a variant of the Spearman rho, which is basically equivalent to the Kendall tau.

Because all of the research participants were well past the age of 18 when they initially completed the BBTS in 2000 (the youngest was about 25 years old at that time), recollections of their childhood experiences should have been more stable than those for events in adulthood, some of which could have been freshly experienced during the threeyear interval between the two administrations. And, indeed, all of the indices of test-retest stability were considerably higher for the childhood events than for those experienced in adulthood; on average, the agreement percentages were $83 \%$ for childhood events as compared with $75 \%$ for those in adulthood. On the other hand, there was no tendency for the women in the sample to have higher indices of stability than the men (the men's values averaged $84 \%$ vs. $82 \%$ for the women with the childhood events and $78 \%$ vs. $72 \%$ with the adult events).

As should be evident from Table 2, any conclusions about the absolute stability of these reported experiences will be highly dependent on the particular type of index used for this purpose. Based on conventional indices of effect size, conclusions based on the percentage agreement and 
the gamma coefficient might be interpreted as indicating considerable test-retest stability, whereas inferences based on the correlation and the kappa coefficients are severely affected by some of these highly skewed response distributions.

\section{Finally, Those Missing Events}

On both versions of our survey, we gave the participants the opportunity to report having experienced "a seriously traumatic event not already covered." As indicated in Table 1, around $20 \%$ of the sample reported having experienced such an event in childhood, and around $40 \%$ reported having experienced such an event in adulthood. In the second survey, we asked participants to describe each of these missing events, and most of the individuals who checked that response option did provide such event descriptions. Many of these "other seriously traumatic" events involved the (non-witnessed) death of, or serious harm to, someone close to the participants. These events included natural and accidental deaths, as well as murder, suicide, and sex abuse. In addition to these traumas by proxy, so to speak, the remaining "other" events included war traumas, marital problems, health problems, and abortion. For future versions of the BBTS, an item assessing death or harm to a close other is strongly recommended. An item specific to war trauma would also be useful, particularly with populations likely to have such exposure.

\section{DISCUSSION}

In this paper, we have described the development of two versions of a survey of potentially disturbing events, and demonstrated the usefulness of this preliminary instrument in examining event stability over time. At this point, we will review our major findings, suggest ways to improve the instrument in future applications, and discuss some limitations on the conclusions that can be drawn from self-report surveys like this one.

\section{Gender Differences in Event Frequencies}

For a number of the events included in our survey, there were substantial gender differences in the self-reported event frequencies. Women reported much higher rates of sexual abuse in both childhood and adulthood, as well as assaults on them perpetrated by someone close to them, especially in adulthood. This finding is consistent with the literature on 
sexual abuse and spousal assault. On the other hand, men reported higher frequencies of witnessing someone not close to them being killed or severely injured, and most strikingly, for being severely attacked themselves by someone not close to them. This finding doubtless reflects men's higher likelihoods of being in physically aggressive situations such as war, gang fights, or violent workplace settings. Men were also more likely to report having experienced serious accidents as adults, but not by such a large margin. Taken together the pattern suggests that women may experience more betrayal-related events than men, but men experience more violent non-betrayal events than women.

Perhaps the most striking illustration of this pattern is revealed in the comparison between the event described as "were yourself severely attacked by someone close" and the same event with "someone not close." Women were almost six times more likely to report having experienced an attack in adulthood by someone close to them than were men; men were over three times more likely to report attacks in adulthood by someone not close to them than were women. To the extent that betrayal-related events are potent for some sorts of psychological impacts whereas other events are potent for other impacts (Freyd, 1999), these gender differences would imply some non-subtle socialization factors operating as a function of gender (DePrince \& Freyd, 2002).

However, it is possible that men and women may not have had exactly the same experience even when they reported having experienced the same event. As an extreme example, if participants are asked whether they had ever experienced "any traumatic event," we would not expect that men and women responding affirmatively would necessarily have the same type of experiences in mind-if for no other reason than the fact that men and women are differentially vulnerable to different types of events.

Although the present descriptions of events were far more specific, it is still possible that self-reports of the same event might be based on quite different experiences, and this may be particularly true for events involving potential sexual abuse. The nature of the relationship between perpetrator and victim may vary substantially as a function of the gender of the victim. For instance, boys may be more likely to be victimized by a close non-parent such as a priest, camp counselor, or coach. Girls may be more likely to be victimized by a parent. If so, the age and duration of abuse are likely to have differed. To the extent that the underlying events are in fact different for males and females, we would expect different patterns of correlations with other variables, which will be the focus of our later reports. 
Nonetheless, even from the evidence provided in this paper, it is possible to refute a widespread conjecture about gender-related reporting biases. Although it has long been assumed that women tend to over-report the occurrence of potentially traumatic events when compared with men, we have shown that there is no such general tendency. Rather there are substantial sex differences in both directions, with women reporting more betrayal-related events and men reporting more interpersonal events not involving someone particularly close to them. This finding has important implications for the victimization literature.

\section{Do Men and Women Interpret the Word "Close" Differently?}

Could the gender effects we have discovered reflect a difference in the way that men and women interpret close relationships, rather than any actual differences in their experiences? For example, do women categorize individuals as "close" whom men categorize as "not close?" We can evaluate this possibility by examining the information obtained in our second survey when we asked for the actual relationship of the perpetrator to the target. Participants were instructed that for each of events 7-12 in Table 1 they should indicate if the "person who did this to you" was (1) Parent or guardian; (2) Other family member; (3) Husband, wife, or romantic partner; (4) Babysitter or nanny; (5) Teacher, doctor, coach, religious counselor, or other professional; (6) Friend or acquaintance; or (7) Stranger. We inspected the responses to these perpetrator categories to determine if the gender effect would be attenuated using the actual specific relationships instead of the broader "close" versus "not close" categories.

We found just the opposite: The gender interaction effects were even larger when perpetrators were classified by their specific relationships with the participants. For example, who gets attacked by whom? Fiftyfive women reported attacks by a spouse or partner, as compared with only four men. In contrast, only three women reported that they had been attacked by a stranger, as compared with 31 of the men. That is, the finding that women are more likely to report assaults perpetrated by someone close to them, and that men are more likely to report assaults perpetrated by someone not close to them, is even clearer when we use the specific relationship categories. Although this finding cannot rule out all types of gender-correlated response biases, it suggests that the most obvious alternative explanation of this type (that men and women interpret close relationships differently) cannot account for the findings. 


\section{The Gender of the Perpetrators}

In our second survey, the participants were also asked to indicate for each event that involved a perpetrator that individual's gender (Male or Female). The vast majority of perpetrators were reported to have been male by both men and women participants. For example, of the perpetrators of physical assaults, 202 of 229 (88\%) were reported to have been males. Of the perpetrators of unwanted sexual contacts, 264 of 290 $(91 \%)$ were reported to have been males. Of the perpetrators of emotional mistreatment, 206 of 282 (73\%) were reported to have been males.

\section{Questions for Future Research on Gender Effects}

We have discovered striking gender differences in the participants' reported exposure to betrayal-related events (that is, events perpetrated by someone close to the participant) versus events that are not as likely to involve betrayal. Do these differences in exposure account for differences in the amount of distress experienced by men and women? For instance, could the higher rates of depression found in female populations be related to the higher rates of betrayal-related events that they apparently experience? Are the much higher rates of male perpetration related to the different traumas to which men are exposed? These questions will be the focus of subsequent reports.

\section{Some Limitations of Our Research}

Throughout this article, we have tried to emphasize the fact that our findings are all based on self-reported experiences, and that these reports are all retrospective in nature. That is, our participants retrospectively reported about their potentially disturbing experiences, and no external corroboration for these events was obtained. It is, therefore, likely that some participants reported having experienced events that did not actually occur and that some participants reported that they had never experienced events when in fact they actually had occurred. Evidence from studies with either a prospective design and/or with independent corroboration of event occurrence would be useful to compare with our current findings.

However, although studies with corroborated samples minimize false positives (reporting events that never actually occurred), they are less able to catch false negatives (believing that no abuse occurred, when in fact it did). In studies involving a prospective design in which individu- 
als with documented abuse histories are later asked to report prior traumas, some failure to report the documented abuse has been found (Goodman et al., 2003; Johnson et al., 1999; Williams, 1994). We thus have good reason to suspect that some of our participants who did not report any exposure to potentially traumatic events had in fact been so exposed. Presumably, there are some number of false positives as well, although it is much more difficult to estimate the percentage of such false reports.

Researchers who have specifically sought to evaluate the accuracy of self-reports of potentially disturbing events have generally found that such reports are not alarmingly inaccurate (e.g., Watt \& Stewart, 2003) nor are inaccuracies obviously correlated with such variables as psychopathology (Brewin, Andrews, \& Gotlib, 1993) or with the availability of the memories over time (Dalenberg, 1996; Williams, 1995). Indeed, as Williams (1995) noted: "In fact, when one considers the basic elements of the abuse, their retrospective reports are remarkably consistent with what had been reported in the 1970s [the time of the documentation of the event]" (p. 662).

A particular problem for interpreting results using our instrument arises if errors, particularly false negatives, are more likely when there are abusive events involving a close other. This may occur because perpetrators of abuse who are parents of the victim or otherwise in a position of power over the victim may have a great deal of control over the day-to-day lives of their victims; this control may enable them to keep the abuse secret, making corroboration and thus validation of memories unlikely. This secrecy and control, and lack of external validation, could make it more likely that victims fail to report the abuse later, or, as predicted by betrayal-trauma theory, the closeness of the perpetrator may add impetus for the victim to remain unaware of the abuse (Cheit, 2003; Freyd, 2003; Freyd, DePrince, \& Zurbriggen, 2001). In support of this possibility, in a major prospective study (see analyses by Freyd, 1996, based on data from Williams, 1994), false negatives (those individuals failing to report documented abuse) were more likely to have been abused by a relative than by a non-relative. Similarly, in a more recent prospective study, Goodman et al. (2003) reported that $80 \%$ of individuals who received maternal support at the time of the criminal investigation disclosed abuse upon later interview, versus $68 \%$ of those who did not receive such support. As Goodman et al. (2003) observed: "Individuals who, as children, felt believed, supported, and legitimated when making their allegations may be more willing or able years later to discuss their victimization" (p. 116). 


\section{SUMMARY}

In conclusion, this paper reports on the development of a new brief measure of exposure to potentially traumatic events, the BBTS, and provides findings from two administrations of the BBTS in an adult community sample. The BBTS includes separate items for events that involve sexual, physical, and emotional mistreatment by someone close, mistreatment by someone not so close, and non-interpersonal events. For each event, respondents indicate the extent of their exposure both prior to and after age 18. Substantial differences between men and women were found for many of these events on both occasions. Many more women than men reported having experienced traumatic events perpetrated by someone close to them, whereas far more men than women reported having experienced traumatic events perpetrated by someone who was not so close. Because the information available in this community sample is so extensive, including the scales from a dozen current personality inventories and many dozens of measures of both mental and physical health, it should be possible to discover some of the associations between potentially traumatic events and a wide assortment of important life outcomes. We invite our readers to join us in this quest.

\section{REFERENCES}

Brewin, C.R., Andrews, B., \& Gotlib, I.H. (1993). Psychopathology and early experience: A reappraisal of retrospective reports. Psychological Bulletin, 113, 82-98.

Carlson, E.B. (1997). Trauma assessments: A clinician's guide. New York: Guilford.

Cheit, R.E. (2003). The limitations of a prospective study of memories for child sexual abuse. Journal of Child Sexual Abuse, 12, 105-111.

Dalenberg, C.J. (1996). Accuracy, timing and circumstances of disclosure in therapy of recovered and continuous memories of abuse. Journal of Psychiatry and Law, 24, 229-275.

DePrince, A.P., \& Freyd, J.J. (2002). The intersection of gender and betrayal in trauma. In R. Kimerling, P.C. Oumette, \& J. Wolfe (Eds.), Gender and PTSD (pp. 98-113). New York: Guilford.

Elliott, D.M. (1997). Traumatic events: Prevalence and delayed recall in the general population. Journal of Consulting and Clinical Psychology, 65, 811-820.

Freyd, J.J. (1994). Betrayal trauma: Traumatic amnesia as an adaptive response to childhood abuse. Ethics \& Behavior, 4, 307-329.

Freyd, J.J. (1996). Betrayal trauma: The logic of forgetting childhood abuse. Cambridge, MA: Harvard University.

Freyd, J.J. (1999). Blind to betrayal: New perspectives on memory for trauma. Harvard Mental Health Letter, 15, 4-6. 
Freyd, J.J. (2001). Memory and dimensions of trauma: Terror may be "all-too-well remembered" and betrayal buried. In J.R. Conte (Ed.), Child sexual abuse: Knowns and unknowns - A volume in honor of Roland Summit. Oakland, CA: Sage.

Freyd, J.J. (2003). Memory for abuse: What can we learn from a prosecution sample? Journal of Child Sexual Abuse, 12, 97-103.

Freyd, J.J., DePrince, A.P., \& Zurbriggen, E.L. (2001). Self-reported memory for abuse depends upon the victim-perpetrator relationship. Journal of Trauma \& Dissociation, 2, 5-17.

Goldberg, L.R. (1999a). A broad-bandwidth, public-domain, personality inventory measuring the lower-level facets of several five-factor models. In I. Mervielde, I. Deary, F. De Fruyt, \& F. Ostendorf (Eds.), Personality psychology in Europe, Vol. 7 (pp. 7-28). Tilburg, The Netherlands: Tilburg University Press.

Goldberg, L.R. (1999b). The Curious Experiences Survey, a revised version of the Dissociative Experiences Scale: Factor structure, reliability, and relations to demographic and personality variables. Psychological Assessment, 11, 134-145.

Goodman, G.S., Ghetti, S., Quas, J.A., Edelstein, R.S., Alexander, K.W., Redlich, A.D., Cordon, I.M., \& Jones, D.P.H. (2003). A prospective study of memory for child sexual abuse: New findings relevant to the repressed-memory debate. Psychological Science, 14, 113-118.

Johnson, J.G., Cohen, P., Brown, J., Smailes, E.M., \& Bernstein, D.P. (1999). Childhood maltreatment increases risk for personality disorders during early adulthood. Archives of General Psychiatry, 56, 600-606.

National Center for PTSD (2006). Website of the The National Center for Post-Traumatic Stress Disorders, US Department of Veteran Affairs. http://www.ncptsd. va.gov/

Norris, F.H. (1992). Epidemiology of trauma: Frequency and impact of different potentially traumatic events on different demographic groups. Journal of Consulting and Clinical Psychology, 60, 409-418.

Norris, F.H., \& Riad, J.K. (1997). Standardized self-report measures of civilian trauma and posttraumatic stress disorder. In J.P. Wilson \& T.M. Keane (Eds.), Assessing psychological trauma and PTSD (pp. 7-42). New York: Guilford.

Watt, M.C., \& Stewart, S.H. (2003). Dyscontrol Experiences Questionnaire: Development, reliability, and validity. Journal of Psychopathology and Behavioral Assessment, 25, 155-165.

Williams, L.M. (1994). Recall of childhood trauma: A prospective study of women's memories of child sexual abuse. Journal of Consulting and Clinical Psychology, 62, 1167-1176.

Williams, L.M. (1995). Recovered memories of abuse in women with documented child sexual victimization histories. Journal of Traumatic Stress, 8, 649-674.

Wilson, J.P., \& Keane, T.M. (2004). Assessing psychological trauma and PTSD, Second Edition. New York: Guilford.

RECEIVED: $10 / 10 / 05$

REVISED: 02/15/05

ACCEPTED: 02/15/05 


\section{APPENDIX}

\section{Personal Experiences}

We hope that you trust us to keep your responses in complete confidence and privacy; this is the reason that we ask you not to include your name on any of our questionnaires. Nonetheless, if you feel uncomfortable answering any of the more intimate questions in this section, just skip them, and go on to the next section. For each item below, please mark one response in the columns labeled "Before Age 18" AND one response in the columns labeled "Age 18 or Older."

BEFORE AGE 18 AGE 18 or OLDER

Have each of the following events happened to you, and if so, how often?

Been in a major earthquake, fire, flood, hurricane, or tornado that resulted in significant loss of personal property, serious injury to yourself or a significant other, the death of a significant other, or the fear of your own death

Been in a major automobile, boat, motorcycle, plane, train, or industrial accident that resulted in similar consequences . . .

Witnessed someone with whom you were very close (such as a parent, brother or sister, caretaker, or intimate partner) committing suicide, being killed, or being injured by another person so severely as to result in marks, bruises, burns, blood, or broken bones. This might include a close friend in combat . .

Witnessed someone with whom you were not so close undergoing a

Witnessed someone with whom you were very close deliberately attack another family member so severely as to result in marks, bruises, blood, broken bones, or broken teeth . ...............

You were deliberately attacked that severely by someone with whom you were very close

You were deliberately attacked that severely by someone with

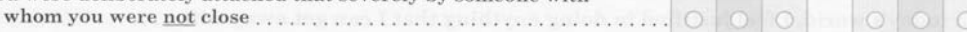

You were made to have some form of sexual contact, such as touching or penetration, by someone with whom you were very close (such as a parent or lover)

You were made to have such sexual contact by someone with whom you were not close

You were emotionally or psychologically mistreated over a significant period of time by someone with whom you were very close (such as a parent or lover)

Experienced the death of one of your own children

Experienced a seriously traumatic event not already covered in any of these question 